safety programs were also associated with better self-reported safety performance of coworkers, crews, and individual workers. Stronger safety programs incorporated activities from all four domains Safety programs that include activities that cover safety of management and worker influence safety performance and safety climate as perceived by the workers.

\section{P.1.09 RISK ASSESSMENT OF EXPOSURE TO FORMALDEHYDE IN UNIVERSITY LABORATORIES}

Alireza Dehdashti. Social Determinants of Health Research Center, Semnan University of Medical Sciences, Semnan, Iran

\subsection{6/OEM-2019-EPI.215}

Objective Formaldehyde is a commonly used chemical in laboratories and has been regarded as a potential hazard to health. Researchers and students who work in medical laboratories have potentially a higher risk of exposure to Formaldehyde. The aim of this study was to determine the risk of exposure to formaldehyde and its health effects in laboratories.

Methods In a cross-sectional survey air samples were collected with charcoal tubes from 23 anatomy, pathology and histopathology laboratories. Personal and time weighted exposures of 102 samples were sampled and analyzed using OSHA method. A questionnaire was distributed to 83 participants engaged in laboratory activities to examine the adverse health effects of formaldehyde.

Results Overall, 92.3\% of the personal exposure levels were higher than the occupational exposure limit. The area concentrations ranged between $0.234 \mathrm{ppm}$ and $3.45 \mathrm{ppm}$ $($ mean $=1.43 \mathrm{ppm}, \mathrm{SD}=0.45)$. Individual exposure levels in respiratory zone ranged $0.219 \mathrm{ppm}$ and $1.96 \mathrm{ppm}$ (mean $=0.573$ ppm, $\mathrm{SD}=0.39$ ). The risk of Formaldehyde exposure levels were higher for researchers and technicians compared to students with a factor of two or three. Participants with a duration of exposure more than 2 years had an increased risk of health symptoms (Hazard ratio $=1.4$; Confidence Interval $=0.8-$ 3.7). Laboratory personnel reported physical fatigue (39.1\%), headache $(32.7 \%)$, breathing problems $(21.1 \%)$, noise irritation (17.4\%), and eye irritation (14.9\%).

Conclusion The concentration levels of Formaldehyde vapor in laboratory indoor air were higher compared to individual exposure levels. Work in laboratories is associated with exposure levels exceeding the recommended exposure limit and an increased risk of health complaints. Preventive measures are required to reduce the emission of airborne formaldehyde and prevalent health-related symptoms in academic medical laboratories.

\section{P.1.11 'CREATION OF CLASSIFICATION MODEL USING MACHINE LEARNING; TO DETECT DYSPHONIA WORK-RELATED'}

${ }^{1}$ Natalia Gilbert, ${ }^{2}$ Rodrigo Assar, ${ }^{2}$ Rodrigo Martinez. ${ }^{1}$ Instituto de Salud Pública de Chile, Santiago, Chile; ${ }^{2}$ Universidad de Chile, Santiago, Chile

\subsection{6/OEM-2019-EPI.216}

The underdiagnosis of occupational disease causes severe damage to the health system. The classification of a disease as a professional is based on the decision on whether the present labor factors are sufficient for the generation of the disease, and this function is carried out by a qualified professional or committee.

Occupational dysphonia is one of the 5 most frequent occupational diseases in Chile, whose condition impact on the labor productivity and the quality of life of the patient. Today there are no unified criteria among the occupational qualification decisión makers to decide on the sufficient of laboral factors of occupational dysphonia disease.

Computerized systems have been developed to support clinical diagnosis decision-making process; among these, Machine Learning methods have been used to simulate the reasoning of the expert from the analysis and identification of complex patterns in large databases, so in this study it is suggested that the creation of a dysphonia classification model is possible employing Machine Learning tools. For this purpose, 103 cases obtained from patients with qualification results cause by dysphonia was analize in relation to the number of variables studied and their distribution for the observation of the characteristics that give identity to the groups studied. Subsequently, different classification models were developed using Machine Learning and the one that presented the best performance was chosen.

Statistical analyzes show that of the 6 models of Machine Learning elaborated, Random Forest was the one that presented the best performance (accuracity $=0.83$ and Kappa value $=0.61$, variables that manage to establish identity to each group represent $26.5 \%$ of the total of studied variables. The results in this work show the potential of the use of computer tools can be useful as a support tool for diagnosis of occupational disease.

\section{P.1.12 PARTICULATE MATTER MEASUREMENT SYSTEM USING LOW COST SENSOR AND INTERNET OF THINGS}

Seunghon Ham, Seong-Kyu Kang. Department of Occupational and Environmental Medicine, Gil Medical Center, Gachon University College of Medicine, Incheon, South Korea

\subsection{6/OEM-2019-EPI.217}

The Internet of Things (IoT) is an important keyword in the Fourth Industrial Revolution. IoT is expected to have a massive impact for human being, but these are still early days. IoT enables these objects to collect and exchange data through the wireless network, such as devices, vehicles, buildings, and other items that include electronic devices, software, sensors, and network connections. IoT is a technology that can be used and expanded in various applications such as industrial, manufacturing, medical and consumer products and so on. This research focuses specifically on the development of measurement systems for particulate matter using IoT technology for occupational as well as environmental settings. Currently, the government is providing the concentration of particulate matter (PM2.5/10) hourly. However, it is difficult to reflect personal exposure because it is installed on the top of a building. Therefore, this study shows development of the particulate matter measuring system using IoT and obtains the personal measurement data of the particulate matter. Also, to provide a further insight, we will also present how particulate matter measurement system works by showing some measurements collected with an experimental testbed deployed in our research group. Still, it is necessary to improve the quality of system and the convergence between pollutant measurement including particulate matter and IoT can provide new 